\title{
Reflets
}

Revue ontaroise d'intervention sociale et communautaire

\section{Coupures dans les services sociaux : impact sur les femmes victimes de violence et leurs enfants}

\section{Carole Giguère}

Volume 3, numéro 1, printemps 1997

Enfance et familles en contexte d'appauvrissement

URI : https://id.erudit.org/iderudit/026154ar

DOI : https://doi.org/10.7202/026154ar

Aller au sommaire du numéro

Éditeur(s)

Reflets : Revue ontaroise d'intervention sociale et communautaire

ISSN

1203-4576 (imprimé)

1712-8498 (numérique)

Découvrir la revue

Citer cet article

Giguère, C. (1997). Coupures dans les services sociaux : impact sur les femmes victimes de violence et leurs enfants. Reflets, 3(1), 113-119.

https://doi.org/10.7202/026154ar

Tous droits réservés (C) Reflets : Revue ontaroise d'intervention sociale et communautaire, 1997
Ce document est protégé par la loi sur le droit d'auteur. L'utilisation des services d'Érudit (y compris la reproduction) est assujettie à sa politique d'utilisation que vous pouvez consulter en ligne.

https://apropos.erudit.org/fr/usagers/politique-dutilisation/ 


\section{Coupures dans les services sociaux: impact sur les femmes victimes de violence et leurs enfants}

\section{Carole Giguère}

Depuis le début des années 1990 et plus concrètement à compter de 1995, les maisons d'hébergement et les associations qui les regroupent font face à une instabilité financière se traduisant par la menace de la survie de certaines d'entre elles et l'insécurité des autres face à leur avenir. L'aide gouvernementale qui a permis la stabilité financière et la croissance des services dans ces maisons n'est plus aussi présente qu'auparavant. Les coupures annoncées par le gouvernement conservateur de Mike Harris au printemps ' 95 tendent vers une nette diminution des services et des ressources communautaires, obligeant les maisons d'hébergement et les organismes communautaires pour femmes victimes de violence à faire plus avec moins d'argent si elles veulent maintenir une qualité de services. Le 21 juillet 1995, le ministère des Services sociaux et communautaires de l'Ontario envoyait un communiqué aux organismes :

[...] Nous réduirons de 21,6\% les prestations d'aide sociale, ce qui entrera en vigueur le $1^{\text {er }}$ octobre prochain. [...] Pour l'exercice 1995-1996, le ministère réduira de 2,5\% les paiements versés aux organismes de services sociaux. En 1996-1997, cette réduction sera portée à $6 \%$ des budgets des organismes. Les réductions commenceront à prendre effet le $1^{\text {er }}$ octobre 1995 (ministère des Services sociaux et communautaires de l'Ontario, juillet 1995). 
Depuis octobre 1995, la «révolution Harris» affecte directement et indirectement les femmes victimes de violence et leurs enfants.

Emphasis on crises and complementary earmarking of funds for programs responding to the immediate needs of safety and emergency health care, meant that funding and energy of front-line workers for follow-up, support programs, analysis and advocacy became a luxury (MacLeod 1994:10).

À l'automne 1995, certaines maisons d'hébergement recevaient un premier avis de fermeture imminente. Le gouvernement Harris venait d'annoncer la fermeture incessante de l'O.A.I.T.H. (Ontario Association for Interval Transition Houses) et l'éventuelle fermeture des maisons d'hébergement de deuxième étape en Ontario. Harmony House, la seule maison de deuxième étape dans l'Est de l'Ontario et située à Ottawa, est toujours menacée par une fermeture incessante. Indirectement, les femmes victimes de violence allaient subir d'autres entraves dans leur décision de mettre fin définitivement à leur situation de violence conjugale. En effet, dans l'édition du 28 mars 1996 du quotidien The Ottawa Citizen, on pouvait lire: Local lawyers refused new legal aid work, puisqu'à compter du $1^{\text {er }}$ avril 1996, l'aide juridique allait subir elle aussi, le couperet de Mike Harris. Ces coupures ont une influence directe sur la décision des femmes victimes de violence de divorcer. Elles n'ont pas les moyens financiers d'entreprendre ces démarches sans appui des services d'aide juridique, comme elles en avaient la possibilité auparavant.

L'aide juridique a, en partie disparu. Plusieurs avocats ne sont plus en affaires, tandis que d'autres refusent maintenant d'offrir des services d'aide juridique. Les frais reliés au dossier de divorce ne sont plus subventionnés par l'aide juridique; résultat: les femmes victimes de violence doivent utiliser les services de médiation et confronter ainsi le conjoint qui les a abusées. Cette situation les retourne dans leur univers de concession et de victimisation. Non seulement elles 
perdent presque toujours mais elles subissent encore une fois l'autorité masculine du système patriarcal, puisqu'il est impossible d'arriver à une entente mutuelle lorsqu'il y a relation abusive (entrevue avec Liette Perron, coordonnatrice de la Maison d'amitié 1996).

When governments find they can't control the alternative services, funds are eventually cut or eliminated. For example, when funds are taken away from women's shelters, the users either go without support or have to resort to the traditional services such as the Salvation Army and the Welfare Department. Nevertheless, despite such setbacks, the number of shelters for abused women has grown steadily from a handful in 1975 to 371 in 1993. Furthermore, women's shelters have begun to widen their response to answer the needs of women who are oppressed because of ethnic background or disability as well as gender (Carniol 1995:129-130).

D'autres services parallèles aux maisons d'hébergement tels que Immigrant and Visible Minority Against Women's Abuse qui offre des services d'interprètes culturels, les services de soins à domicile, qui offrent des services d'aide infirmier et de soutien à domicile aux femmes malades et handicapées ont, eux aussi, subi des coupures, restreignant leur capacité de desservir les femmes en maison d'hébergement. L'accessibilité aux services et aux maisons d'hébergement pour les femmes de groupes ethniques, de toutes les régions et de toutes les classes sociales est remise en question après tant d'efforts des groupes de femmes depuis plusieurs années.

Presently, the government is concentrating on the cost of service per client. This involves a formula, that reduces the client to a dollar figure. One of the results of this formula, is the rate of occupancy. Shelters must have a minimum of $75 \%$ of its beds occupied, it is threatened with the withdrawal of its funding. This affects predominantly rural shelters, which rarely have a $75 \%$ occupancy rate. In the last year, many rural shelters 
have had their funding revoked and were forced to close (Peltier, shelter director in Hodge, 1995:15.)

Une cinquantaine de maisons d'hébergement, particulièrement les maisons de deuxième étape, devraient fermer leurs portes en Ontario en 1996 (Le Droit 1995: 2). Ce même quotidien relevait les données de Statistique Canada montrant que plus de 85000 femmes et enfants ont eu recours aux services des maisons d'hébergement pour femmes battues entre mai 1994 et mai 1995, soit une augmentation de 3000 personnes en 1995 en plus de la création de 34 nouvelles maisons d'hébergement au cours de ces trois années (13 décembre 1995: 2). Ces maisons ont reçu une aide financière du gouvernement néo-démocrate de Bob Rae qui s'est poursuivie avec le nouveau gouvernement conservateur de Mike Harris. Malgré cela, les programmes sociaux subissent des coupures depuis 1993. Ces coupures sont de l'ordre de $20 \%$ au total pour la Maison d'amitié (Entrevue avec Liette Perron, coordonnatrice de la Maison d'amitié) D'autres coupures sont à prévoir pour les prochaines années.

Les mesures annoncées aujourd'hui ne constituent que le début de la tâche que le gouvernement s'est fixée et qui vise à réduire les dépenses provinciales. Dans les mois et les années à venir, il y aura des décisions encore plus difficiles à prendre (ministère des Services sociaux et communautaires émis le 17 juillet 1995 à l'intention des intervenants et intervenantes)

Le gouvernement Harris avait pourtant dit, à son entrée en juin 1995 qu'il maintiendrait les services essentiels. On peut donc croire que les services pour les femmes victimes de violence et leurs enfants ne sont pas essentiels, pas plus qu'il est essentiel que 85000 femmes et enfants soient en sécurité au pays à chaque année. Il s'agit en effet du nombre de personnes ne pouvant être admises en maison d'hébergement, faute d'espace disponible, voilà «l'essentiel» à considérer. Le tableau sommaire des statistiques de la Maison d'amitié aide à mieux comprendre la réalité des maisons d’hébergement durant la période de 1993-1995. 
Tableau 1

Statistiques générales de la Maison d'amitié

\begin{tabular}{lrrr}
\hline Année : & $\mathbf{1 9 9 3}$ & $\mathbf{1 9 9 4}$ & $\mathbf{1 9 9 5}$ \\
\hline Nombre d'appels reçus : & 1021 & 1065 & 1313 \\
- Relation d'aide : & 332 & 522 & 574 \\
Demandes d'hébergement : & 507 & 689 & 724 \\
- Admissions : & 118 & 127 & 126 \\
- Refus : & 389 & 562 & 598 \\
\hline Aide sociale à l'arrivée: & $44 \%$ & $52 \%$ & $47 \%$ \\
Aide sociale au départ: & $63 \%$ & $68 \%$ & $71 \%$ \\
Départs sans conjoint: & $79 \%$ & $72 \%$ & $78 \%$ \\
Retours avec ex-conjoints: & $15 \%$ & $21 \%$ & $14 \%$ \\
Durée moyenne du séjour (jours) : & 45 & 46 & 60 \\
\hline
\end{tabular}

N.B. Le budget annuel de la Maison d'amitié a été réduit de $21,6 \%$ au cours de ces trois années.

«Si on ne peut

affirmer avec

exactitude quelles sont

les répercussions

directes des coupures

budgétaires du

gouvernement de

l'Ontario sur la situation des femmes

durant leur séjour en

maison d'hébergement, on peut cependant constater que les services offerts répondent à une infime partie de la demande.»
Grâce à ce tableau statistique sommaire, on peut constater que le niveau de revenu des familles diminue considérablement durant leur séjour à la Maison d'amitié. Plus de femmes doivent dépendre de l'aide sociale durant et après leur séjour en maison d'hébergement. Nous sommes en mesure d'observer que le nombre d'appels téléphoniques ainsi que le nombre de demandes d'hébergement croissent sans cesse. Cependant, le nombre de places disponibles demeure toujours le même. Si on ne peut affirmer avec exactitude quelles sont les répercussions directes des coupures budgétaires du gouvernement de l'Ontario sur la situation des femmes durant leur séjour en maison d'hébergement, on peut cependant constater que les services offerts répondent à une infime partie de la demande.

Malgré l'évolution de la situation de la femme en général et des services dispensés aux femmes et enfants victimes de violence conjugale, la situation globale est toujours aussi inquiétante que dans les années 1970. Malgré toutes ces années de changement et de reconnaissance sociale, la majorité des femmes victimes de violence et leurs enfants n'a toujours pas accès aux services qu'elles seraient en droit de recevoir. 
Aujourd'hui encore, les maisons d'hébergement sont la ressource ultime pour les femmes victimes de violence et leurs enfants. Elles accueillent annuellement plus de 85000 femmes et enfants à travers le Canada. Bien qu'elles ne suffisent pas à la tâche, ne pouvant répondre qu'à une demande d'hébergement sur cinq, les gouvernements actuels réduisent considérablement leur appui financier. La conscience sociale trouve-t-elle ses limites dans l'aide gouvernementale? Il serait permis de penser que plus les études démontrant l'ampleur du problème se multiplient, plus les solutions et l'aide gouvernementale augmenteraient... Or, depuis quelques années, la réalité est toute autre. La survie des maisons d'hébergement est en jeu. D'une part on reconnait leur importance et, d'autre part, on assiste à leur disparition graduelle.

\section{Bibliographie}

BEAUDRY, M. (1984). Les maisons des femmes battues au Québec - groupe d'analyse des politiques sociales, Montréal, Éditions Saint-Martin.

CANADA, Ministère de la Justice (1985). La loi sur les agressions sexuelles au Canada - Une évaluation: les nouvelles infractions en matière d'agression sexuelle: Questions juridiques d'actualité, Rapport no.2, Direction de la politique des programmes et de la recherche, Ottawa, Section des programmes et de la recherche.

CANADA, SANTÉ ET BIEN-ÊTRE SOCIAL (1992). Maisons de transitions et d'hébergement pour femmes victimes de violence au Canada, Ottawa, Ministère des approvisionnements et services.

CANADA, SANTÉ ET BIEN-ÊTRE SOCIAL (1991). Centre national d'information sur la violence dans la famille - La violence conjugale et ses conséquences sur les enfants, mars, Ottawa, Ministère des approvisionnements et services.

CANADA, SANTÉ ET BIEN-ÊTRE SOCIAL (1990). Child Sexual Abuse Initiative: Summaries of Funded Projects (1986-1989), Ottawa, Ministère des approvisionnements et services.

CANADA, SANTÉ ET BIEN-ÊTRE SOCIAL (1989). Espoirs et déceptions dans le domaine des femmes battues : progrès, dilemmes et perspectives d'intervention, Ottawa, Ministère des approvisionnements et services.

CANADA, SANTÉ ET BIEN-ÊTRE SOCIAL (1986). Étude d'un refuge de la deuxième étape pour les femmes battues et leurs enfants, Ottawa, Ministère des approvisionnements et services.

CANADA, Statistique (1995). Enquête sur les maisons d'hébergement pour femmes violentés, 1994-1995, Tableaux des données 1-20, Ottawa, Ministère des approvisionnements et services.

CARNIOL, Ben (1995). Case critical: Challenging social services in Canada, Toronto, Between the lines, $3^{e}$ édition.

HODGE, Anne (1995). Shelters for abused women: Past, present and futur, travail présenté dans le cadre d'un cours au service social à l'Université Carleton. 
LE DROIT, Journal (1996). TURBIDE, Mathieu et JOANISSE, Marc André, Enfant battu à mort, édition du 24 août, 1996, 1.

LE DROIT, Journal (1995). LEMIEUX, Julie Maisons, édition du 20 décembre, 1995, 1-2.

LE DROIT, Journal (1995). P.C.Toronto Plus de personnes ont recours aux maisons pour femmes battues, éditon du 13 décembre, 1995, 2.

LE DROIT, Journal (1995). LEMIEUX, Julie Maison Harmony se fait couper ses vivres, édition du 4 octobre, 1995, 2.

LE DROIT, Journal (1994) PRESSE CANADIENNE À la défense des enfants, édition du 22 novembre, 1994, 14.

MacLEOD, Linda (1994). Understanding and Charting our Progress Toward the Prevention of Women's Abuse, Ottawa, National Clearing House, Ministry of Supply and Services Canada.

MacLEOD,Linda (1987). Pour de vrais amours... Prévenir la violence conjugale, Conseil consultatif canadien sur la situation de la femme, Ottawa, Ministère des approvisionnements et services.

NATIONAL CLEARING HOUSE (1995). Inventory of Residental Services for Battered Women, Ottawa, Ministry of Supply and Services Canada.

OTTAWA CITIZEN (1996). Branswell, Brenda Local Lawyers Refused New Legal Aid Work, éditon du 28 mars 1996, 1-2.

PRICE, L. (1991). «La violence en héritage — Étude des liens entre l'agression sexuelle à l'égard des enfants et les voies de fait envers les conjointes», Vis-à-vis, Bulletin national sur la violence familiale, Conseil canadien de développement social, 9, 2-7.

URSEL, Jane (1992). Private Lives, Public Policy, - 100 Years of State Intervention in the Family, Toronto, Women's Press.

WALKER, Gillian (1990). Family Violence and the Women's Movement - The Conceptual Politics of Struggle, Toronto, University of Toronto Press.

\section{Références}

1. E.O.A.I.T.H. (East Ontario Association Interval Transition Houses), procès-verbaux de 1977 à 1995.

2. MAISON D'AMITIÉ, procès-verbaux de 1977 à 1995 , documentation interne et formulaires des programmes pour enfants

3. O.A.I.T.H. (Ontario Association Interval Transition Houses), procès-verbaux de 1977 à 1995. 